\title{
The wrist of the formula 1 driver
}

Emmanuel H Masmejean, Hervé Chavane, Alain Chantegret, Jean-Jacques Issermann, Jean-Yves Alnot

\begin{abstract}
Objectives-During formula 1 driving, repetitive cumulative trauma may provoke nerve disorders such as nerve compression syndrome as well as osteoligament injuries. A study based on interrogatory and clinical examination of 22 drivers was carried out during the 1998 formula 1 World Championship in order to better define the type and frequency of
\end{abstract} these lesions.

Methods-The questions investigated nervous symptoms, such as paraesthesia and diminishment of sensitivity, and osteoligamentous symptoms, such as pain, specifying the localisation (ulnar side, dorsal aspect of the wrist, snuff box) and the effect of the wrist position on the intensity of the pain. Clinical examination was carried out bilaterally and symmetrically.

Results-Fourteen of the 22 drivers reported symptoms. One suffered cramp in his hands at the end of each race and one described a typical forearm effort compartment syndrome. Six drivers had effort "osteoligamentous" symptoms: three scapholunate pain; one medial hyperpcomression of the wrist; two sequellae of a distal radius fracture. Seven reported nerve disorders: two effort carpal tunnel syndromes; one typical carpal tunnel syndrome; one effort cubital tunnel syndrome; three paraesthesia in all fingers at the end of a race, without any objective signs.

Conclusions-This appears to be the first report of upper extremity disorders in competition drivers. The use of a wrist pad to reduce the effects of vibration may help to prevent trauma to the wrist in formula 1 drivers.

(Br J Sports Med 1999;33:270-273)

Keywords: wrist; trauma; cumulative trauma; nerve compression; ligamentous distension; racing drivers

In the literature, the incidence of wrist and hand injuries in the general sporting population is estimated to be about $25 \%$. $^{1}$ In weight lifting for instance, wrist lesion occurs in $12 \%$ of lifters. ${ }^{2}$ These injuries can be subdivided into four categories: overuse, nerve (and vascular), traumatic, and the weight lifting injuries. ${ }^{1}$ Weight lifting lesions are more specific to gymnasts. The other three kinds can be observed in other sports where wrists and hands are involved, such as competitive driving. In the formula 1 driver, cumulative microtrauma may produce different pathologies at the level of the upper extremities, such as hand-arm vibration syndrome. ${ }^{3}$ It can be exhibited as nerve disorders, such as nerve compression as part of an overuse syndrome, or osteoarticular lesions. Nerve disorders of the upper extremities are in particular represented by carpal tunnel syndrome at the wrist and by cubital tunnel syndrome at the elbow. Osteoligamentous lesions of the wrist, by progressive distension, are mainly represented by scapholunate ligament distension, but triquetrolunate ligament injuries as well as triangular fibrocartilage complex lesions may also be evoked.

For the formula 1 driver, the frequency and intensity of vibration are such that they may provoke these lesions. We have carried out an epidemiological study based on interrogation and a bilateral clinical examination of the wrists and hands of formula 1 drivers. The goal was to evaluate the type and frequency of such injuries in order to propose preventive measures.

\section{Subjects and methods}

SUBJECTS

During the 1998 French Grand Prix on the Magny-Cours track, 22 formula 1 drivers from 12 different nations were clinically examined by the same independent doctor (EHM). They comprised 19 drivers taking part in the current World Championship and three former formula 1 drivers, who were still driving in other categories (rallying, endurance, etc). Each driver had raced in a mean of 63 formula 1 Grand Prix (range 7 to 176).

The mean age of the drivers was 30.7 years: 28.1 years for the drivers in the current championship and 37.3 years for the three former drivers.

Twenty drivers were right handed and two were left handed.

\section{METHODS}

Questioning established medical and surgical history and other sports played, especially those involving the upper extremities-for example, tennis and golf. Nervous symptoms were also investigated such as paraesthesia and diminishment of sensitivity, and also osteoligamentous symptoms such as pain, with specification of the location (ulnar side, dorsal aspect of the wrist, snuff box) and the effect of the wrist position on the intensity of the pain. The interrogation also researched wrist instability and/or "clunk".

The clinical examination was carried out bilaterally and symmetrically. The basic rules of physical examination of a patient, namely, appearance, palpation, movement, and stress are especially important at the wrist level. ${ }^{4}$ The range of motion of the digits and the wrist in 


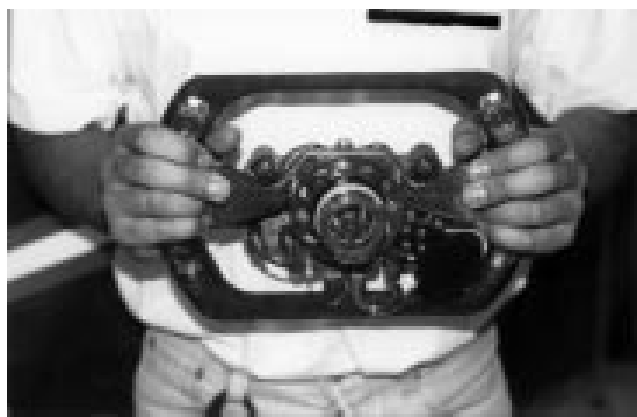

Figure 1 Back view of the bear hand grip on the steering wheel. Note the semiautomatic gear change which is controlled with the long finger on the right hand.

flexion-extension as well as in prosupination was noted. Palpation of the joints identified areas of tenderness, which were related to the intercarpal intervals - that is, the spaces between the scaphoid and the lunate, and the lunate and the triquetrum. The Kirk Watson test $^{5}$ assessed scapholunate instability. The Reagan shuck test was used to look for peritriquetral instability. ${ }^{6}$ Finally a midcarpal instability sign was looked for.

The mobility of the thumb column was evaluated using the opposition scale of Kapandji.

Grip strength was then measured with a grip dynamometer. The key pinch, which evaluates the thumb-index finger pinch, was measured with a special dynamometer.

Sensitivity of the tips of the thumb, the index finger, and the little finger was assessed with Semmes-Weinstein monofilaments. ${ }^{8}$

\section{Results}

QUESTIONING AND CLINICAL EXAMINATION

All the drivers participated in other sports (running, swimming, body building) as training or entertainment. Ten of the 22 drivers played a sport that involved the upper extremities - for example, tennis, squash, and golf.

Among the medical histories, we noted the following traumas, the causes of which were not restricted to motor racing: one polytrauma with a trauma of the pelvic belt and both lower limbs; one fracture of the acetabulum; three leg fractures (including one bilateral case complicated on one side by a compartment syndrome); one bilateral knee fracture and one knee anterior cruciate ligament tear; three ankle fractures (one bilateral); three clavicle fractures; one elbow fracture and one elbow dislocation; one forearm fracture; three wrist fractures; one dorsal ganglion cyst of the wrist (endoscopic removal); two carpal scaphoid fractures.

Only eight drivers did not report any problems with the wrists or hands. All drivers who raced before 1991 complained of palm irritation after each race resulting from using the gear change lever on the right hand side. Since the 1991-1992 season, when a semiautomatic gear change on the steering wheel was introduced (fig 1), none of the drivers have reported pain at the base of the palm.
Tendinomuscular symptoms

Two drivers complained of muscular pain. One described cramps in the hand itself at the end of each Grand Prix. One former formula 1 driver, who still competes in long distance races, described a typical forearm effort compartment syndrome; he also complained of a bilateral effort cubital tunnel syndrome (see below). No tendinopathies or tenosynovitis were observed.

\section{Osteoarticular symptoms}

Six drivers reported such symptoms: three involved the scapholunate ligament, two were sequellae of a distal radius fracture, and one involved hypercompression of the medial compartment of the wrist.

One driver described a typical syndrome of hypercompression of the medial compartment of his non-dominant left wrist. The pain was classically reproducible on ulnar deviation of the wrist. This experienced driver said that these dorsoulnar pains were systematic at the end of each Grand Prix, with a maximum of discomfort after the Italian Grand Prix at Monza, where there are many chicanes (zigzags).

The driver who had had a dorsal ganglion cyst removed previously under endoscopy still suffered dorsal pains after the effort of driving. During the clinical examination, we found pain at the level of the scapholunate ligament and a diminution of range of motion in flexionextension $\left(150^{\circ} v 170^{\circ}\right)$.

Finally, two drivers reported bilateral dorsal pains around the scapholunate ligament space after each Grand Prix. In the clinical examination, there was only tenderness to palpation or subjective complaints and pains without any other objective symptoms.

No drivers tested positive in the scapholunate (Kirk Watson test) or triquetrolunate (Reagan test) tests, nor was any midcarpal instability observed.

One former driver who had had a right wrist fracture complained of dorsal effort pain in his wrist, with the range of motion in flexionextension limited to $70^{\circ}$. Another former driver with a previous right dominant wrist fracture had dorsal effort pain without any objective sign on clinical examination except for a diminution of grip strength $(56 v 58 \mathrm{~kg})$.

\section{Nervous symptoms}

Seven drivers reported paraesthesia in the fingertips. Symptoms were always bilateral. Two drivers described paraesthesia in the thumb and index finger after each race, evoking a subjective form of an effort carpal tunnel syndrome, without any objective sign in the clinical examination. Three drivers had paraesthesia in all the fingertips at the end of each Grand Prix, also with no objective symptoms in the examination. One former driver described a typical carpal tunnel syndrome, with pins and needles at night and an irritative sign of the median nerve at the wrist (Tinel's sign). Another former driver had bilateral effort cubital tunnel syndrome after each race, with paraesthesia in the ring and little fingers. In the 


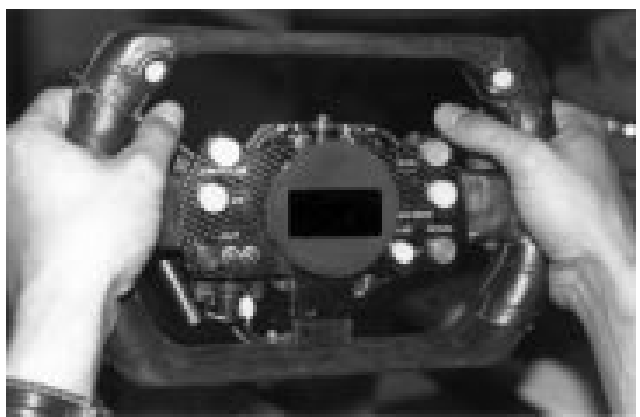

Figure 2 Front view of the bear hand grip. Note the hypertrophy of the lateral thenar muscle and the adaptation of the steering wheel diameter by the use of a grip to avoid muscular symptoms such as cramps.

clinical examination, there was a sharp irritative sign of the ulnar nerve at the level of the flexor carpi ulnaris arcade at the elbow.

No drivers reported subjective diminution of sensitivity of the fingertips.

OBJECTIVE VALUES

Range of motion of the digits

All the drivers had the full range of motion for all the fingers, except the thumb, in extension as well as on making a fist. For the thumb, 16 drivers presented full bilateral opposition (10/10 on Kapandii's scale). For the remaining six, the opposition was $9 / 10$.

Range of motion of the elbow and wrist

At the wrist, the mean flexion was $87.5^{\circ}$ for the dominant hand and $89.8^{\circ}$ for the nondominant hand. Mean extension was found to be $82.5^{\circ}$ for the dominant side and $85^{\circ}$ for the opposite side. Mean pronation was $87.2^{\circ}$ for the dominant side and $88.4^{\circ}$ for the opposite side. Mean supination was $87.7^{\circ}$ on both sides.

Strength

The mean grip strength, evaluated with a Jamar dynamometer, was 53.9 (range 41.5-67) kg for the dominant hand and 50.6 (range 41.5-67) $\mathrm{kg}$ for the non-dominant hand. The key pinch strengths between the thumb and index finger were 10.8 (range 8-12.5) kg for the dominant hand and 9.2 (range 7-12) $\mathrm{kg}$ for the non-dominant hand.

Sensitivity measured with monofilaments

Four drivers had a value of 2.36 for all fingertips. Two drivers had a value of 2.36 for all fingertips of the dominant hand and 2.44 for all fingertips of the non-dominant hand. For one driver, it was the converse. Four drivers presented a value of 2.44 for all fingertips on both hands. One driver presented a value of 2.83 for all fingertips. The 10 remaining drivers had normal sensitivity of between 2.44 and 2.83 on different fingertips.

\section{Discussion}

If the present discovery of nerve and osteoligamentous disorders in this group of high level drivers is predictable, the frequency of such lesions can be considered important. No previous papers have been found in the literature that mention upper extremity problems of racing drivers.
The discovery in two drivers of "muscular" disorders after a race does not appear to us to be a specific problem. In fact, the effort compartment syndrome reported was found in a former driver who is now involved in long distance races (rallying). The quasicomplete disappearance of symptoms such as cramps appears to be due to better adaptation of the wheel diameter to the driver's hand using a rubber grip as used for tennis players (fig 2). Although there are many reports of soft tissue injuries incurred in other sports, ${ }^{9} 10$ we did not observe any in this group of racing drivers. We also found no cases of tendinitis or synovitis, such as is found in players of racket sports, repetitive motion of the wrist in extreme positions contributing to this condition.

Nerve disorders in sportsmen are now well known as part of cumulative trauma disorders, especially in cyclists ${ }^{1}$ and rock climbers. ${ }^{11}$ For the formula 1 driver, we found, like Weinstein and Herring, ${ }^{12}$ that sports associated neurogenic syndromes are usually incomplete, without severe motor or sensory deficits, with typically subjective complaints of pain or vague sensory disturbance. In our series, unfortunately it was not possible to carry out electrodiagnostic testing. Although some authors recommend the systematic use of an electromyogram in injured sportsmen, ${ }^{13}$ we think that an electromyogram and nerve conduction velocity test would only need to be performed on our two patients with nerve compression syndrome with objective symptoms. On the one hand, the absence of electromyogram and nerve conduction velocity abnormalities is not unusual in sportsmen. ${ }^{12}{ }^{14} \mathrm{On}$ the other hand, with regard to hand-arm vibration syndrome, Dasgupta and Harrison ${ }^{3}$ found that in miners the mean motor nerve conduction velocity in the vibration exposed group was significantly decreased. It would be worth while, with hand-arm vibration syndrome of a mild degree, investigating further with a cold challenge test and perhaps thermography after a practice session. We consider that the first preventive treatment should be the use of a palmar wrist pad, as silicone rubber splinting is a well known treatment of sports related hand and wrist injuries. ${ }^{1516}$ Such a pad should partially absorb the vibrations and prevent, or eventually reduce, the paraesthesia that appears during effort.

Pain at the dorsal aspect of the wrist is often attributed to progressive distension of the scapholunate ligament as the result of repetitive trauma, especially in boxers and volleyball players. ${ }^{17}$ The problem in sportsmen is to define which paraclinical examination is required. ${ }^{18}$ We consider that, in the case of symptomatology evoking scapholunate distension due to cumulative trauma, plain $x$ ray examination of the wrists, with anteroposterior and lateral views, is the first step required. To complement this, it is also important to obtain dynamic anteroposterior $x$ ray pictures in ulnar and radial deviation, and a clenched fist view. In the case of a clinical syndrome of hypercompression of the medial aspect of the wrist, an arthro computed tomography or magnetic 
resonance imaging scan can be performed after the $x$ ray examination. Arthroscopy remains interesting as a supplementary aid to diagnosis, and of course as a treatment with the aim of facilitating a rapid return to sport. ${ }^{1}$ As for gymnasts, arthroscopy can be particularly pertinent for triangular fibrocartilage complex injury. ${ }^{19}$

As for nerve disorders, the use of a wrist pad may help to prevent and/or reduce osteoarticular symptoms. It is important to note that a wrist pad would only be useful if it did not limit the range of motion of the wrist required during a competition, which seems to be a realistic goal for formula 1 drivers.

With regard to the objective results, we observed a diminution of the range of motion in all sectors except supination on the dominant side. ${ }^{20}$ It is logical that the musculature of the dominant hand limits this range of motion by a few degrees. Eight drivers who exhibited a limitation of the thumb column opposition at 9/10 showed hypertrophy of the lateral thenar muscles through gripping the steering wheel (fig 2). In all the drivers, sensitivity of the fingertips could be considered normal.

\section{CONCLUSION}

The extent of nerve injuries and/or osteoligamentous disorders resulting from cumulative trauma has been measured for the first time in formula 1 drivers. In our series, $63 \%$ (13 of 22) of the drivers reported problems, either ligamentous progressive distension or nerve disorders presenting essentially as effort compressive syndromes. However, this is a purely clinical study, and a paraclinical examination will be required to define more precisely the pathoanatomy of the lesions. The use of a wrist pad could reduce the consequences of the vibrations at the level of the wrist.
The authors wish to thank Professor Sid Watkins, Medical Delegate for the Fédération Internationale de l'Automobile.

1 Howse C. Wrist injuries in sport. Sports Med 1994;3:16375.

2 Konig M, Biener K. Sport-specific injuries in weight lifting. Schweizeriche Zeitschrift für Sportmedzin 1990;38:25-30.

3 Dasgupta AK, Harrison J. Effects of vibration on the handarm system of miners in India. Occup Med (Oxf) 1996;46: $71-8$.

4 Stanley J. The examination, imaging and investigation of wrist instability. In: Büchler U, ed. Wrist instability. London: Martin Dunitz, 1996:83-96.

5 Watson HK, Black DM. Instabilities of the wrist. Hand Clin 1987;3:103-11.

6 Reagan DS, Linscheid RL, Dobyns JH. Lunotriquetral sprains. F Hand Surg [Am] 1984;9:502-14.

7 Kapandii A Clinical scale for opposition and contraKapandji A. Clinical scale for opposition and contra-
opposition of the thumb. Annals of Hand Surgery 1986;5: opposition.

8 Bell-Krotoski JA. Light-touch-deep pressure testing using Semmes-Weinstein monofilaments. In: Hunter JM, Schneider LH, Mackin EJ, et al, eds. Rehabilitation of the hand, 3rd ed. St Louis: CV Mosby, 1990:585-93.

9 Osterman AL, Moskow L, Low DW. Soft-tissue injuries of the hand and wrist in racquet sports. Clin Sports Med 1988; 7:329-48.

10 Halikis MN, Taleisnik J. Soft-tissue injuries of the wrist. Clin Sports Med 1996;15:235-59.

11 Holtzhausen LM, Noakes TD. Elbow, forearm, wrist and hand injuries among sport rock climbers. Clin f Sport Med 1996;6:196-203.

12 Weinstein SM, Herring SA. Nerve problems and compartment syndromes in the hand, wrist and forearm. Clin Sports Med 1992;11:161-88.

13 Mosher JF, Peripheral nerve injuries and entrapments of the forearm and wrist. In: Pettrone FA, ed. Symposium on upper extremity injuries in athletes. St Louis: American Academy Orthopaedics Surgeons/CV Mosby, 1986:174.

14 Aulicino PL. Neurovascular injuries in the hands of athletes. Hand Clin 1990;6:455.

15 Canelon MF. Silicone rubber splinting for athletic hand and wrist injuries. 7 Hand Ther 1995;8:252-7.

16 Canelon MF, Karus AJ. A room temperature vulcanizing silicone rubber sport splint. Am f Occup Ther 1995;49:2449 .

17 Masmejean E, Dutour O, Touam C, et al. Bilateral SLAC wrist: an unusual entity. Report of a 7000 year-old prehistoric case. Annals of Hand Surgery 1997;16:207-14.

18 Belhobek GH, Richmond BJ, Piraino DW, et al. Special diagnostic procedures in sports medicine. Clin Sports Med 1989;8:517-40

19 De Smet L, Claessens A, Fabry G. Gymnast wrist. Acta Orthop Belg 1993;59:377-80.

20 Mathiowetz V, Kashman N, Volland G, et al. Grip and pinch strength: normative data for adults. Arch Phys Med Rehabil 1985;66:69-72.

\section{Take home message}

Nerve injuries and/or osteoligamentous disorders of the wrist resulting from cumulative trauma have been shown in formula 1 drivers. In our series, $63 \%$ reported problems, either ligamentous progressive distension or nerve disorders mainly presenting as effort compressive syndromes. The use of a wrist pad could reduce the consequences of the vibrations experienced by these drivers at the level of the wrist. 